\title{
The Use of Generalized Additive Model (GAM) To Assess Fish Abundance and Spatial Occupancy in North-West Bay of Bengal
}

\section{Bandanadam Swathi, Swarnalatha. V, Venkatesh Jogu}

Master of Technology in Geoinformatics and Surveying Technology, Jawaharlal Nehru Technological University Hyderabad, Telangana, India

\begin{abstract}
The remote sensing data, such as sea surface temperature \& chlorophyll concentration obtained from various satellites are utilized by Indian National Centre for Ocean Information Services (INCOIS) to provide Potential Fishing Zone (PFZ) advisories to the Indian fishing community which plays a vital role in national GDP. The data on Sea Surface Temperature (SST) is retrieved regularly from thermal-infrared channels of NOAA-AVHRR and chlorophyll concentration (CC) from optical bands of Oceansat-II and MODIS Aqua satellites for the identification of Potential Fishing Zones (PFZ) in Indian water. PFZ information has certain limitations, such as it can't predict the type of fish available in the notified fishing zone. In this dissertation, I have worked towards the development of short-term Hilsa shad predictive capabilities in a sustainable way. An effort has been taken to categorize all essential biological, environmental and climatic signals that have a direct or indirect impact on the Hilsa shad distribution. Remote sensing, ocean biogeochemical modelling, and statistical modelling approach have gained an increasing importance to study the marine ecosystems as-well-as for understanding the dynamics of the oceanic environment. Shad habitat has been studied from the geo-tagged fish catch data and oceanic/ecological indicators as predictor variables. For short-term prediction, the variables have been derived from a biophysical model, configured at INCOIS, using Regional Ocean Model System (ROMS) and remote sensing data. Using generalized additive model (GAM) Catch per Unit Effort (kg h-1) has been calculated as a response variable. Probability maps of predicted habitat with no fishing zone information have been generated using geographic information system.
\end{abstract}

Keywords : Remote Sensing, Potential Fishing Zones, Chlorophyll-A Concentration, Sea Surface Temperature.

\section{INTRODUCTION}

The abilities to develop satellite remote detecting innovation, joined with traditional information gathering strategies, give a capable instrument to the proficient and savvy administration of living assets. Varieties in maritime condition assume a key part in deciding the common dissemination of fish stocks. Laurs et al. (1984) utilized NOAA-AVHRR and Coastal Zone Color Scanner (CZCS) information to comprehend the circulation of Albacore tuna in the waters of California. They found that Albacore fish had a tendency to be moved in the region of temperature fronts on the hotter side the water. Later Arone (1987) utilized bunching method to group distinctive water masses and to describe the connection between the satellite-inferred Sea Surface Temperature (SST) and fish distribution. Solanki et al. (1988) utilized Indian Remote Sensing Satellite ModulerOpto-electronic Scanner (IRS-MOS) and NOAA-AVHRR information to portray the connection between the physical and biological factors. They presumed that the chlorophyll (Chl) and sea surface temperature has conversely 
corresponded. Synergistic investigation of satellitedetermined CHL and SST highlights were completed by Solanki et al. (2001). They found that CHL and SST highlights match at a few areas demonstrating a nearby coupling amongst biological and physical parameters. Potential Fishing Zones (PFZ) are delineated from SST and CHL images and provided operationally as livelihood information to the marginal fishing community of India.

\section{OBJECTIVES}

As with the existing method of PFZ advisory generation can't provide information on the type of fish available in the Potential Fishing Zones, this study aims to illustrate how remotely sensed and modeled oceanic variables and fishing operations data can be used to predict suitable habitat of small pelagic fishery resources, such as Hilsa shad using GIS and Generalised Additive Model (GAM). Also, how the habitat suitability modelling approaches can be utilized in forecasting the fishing grounds of Hilsa shad.

\section{LITERATURE REVIEW}

\subsection{PFZ:}

Solanki et al (2005) built up a satellite-based potential Fishing Zone (PFZs) advisory framework created utilizing incorporation of Ocean Color Monitor (OCM) inferred Chl and NOAA-AVHRR determined SST. Co-enrolling these two information sorts, an ace advisory was generated utilizing ocean features, such as eddies, fronts, and gyres situated on both the images. Validation of the PFZ estimates was done amid the years 1999-2002 utilizing the fish Catch Per Unit Effort (CPUE) data collected from Fishery Survey of India's vessels. Base trawl gets and its percent commitment by species was resolved from $30 \mathrm{~m}$ to $100 \mathrm{~m}$ profundity extend.
Choudhury et al (2007) have advanced an 'integrated Potential Fishing Zone (IPFZ)' map where the facts on capabilities which includes fronts, eddies, gyres, currents and upwelling derived from satellites were included from geo-coded co-registered CHL and SST pics. Fishery Survey of India (FSI) fishing vessels of Visakhapatnam base became used to validate the concerned IPFZs. The capture consistent with the unit attempt CPUE becomes computed taking total trap divided through actual fishing hours. The validation shows that the CPUE is high in the IPFZ regions compared with an environment. In the maximum of the cases, greater than an order growth in fish catch is observed within the IPFZ notified regions.

Zainuddin et al (2011) described the short-term relationship between skipjack tuna and oceanographic conditions and to visualize the predicted high catch areas, remotely sensed satellite based-oceanographic SST and CHL together (Aqua / MODIS) from fisheries data were used. Results indicated that the highest skipjack CPUEs were mainly found in the SST region of $29.0-31.5^{\circ} \mathrm{C}$. The preferred ranges provide a good indicator for initially detecting potential skipjack fishing grounds.

Lan et al (2012) used cloud-free Advanced Microwave Scanning Radiometer for the Earth Observing System (AMSRE) SST and daily set longline fishery data, to study the relationship between albacore (ALB) fishing grounds and thermal conditions in the southern Indian Ocean. SST and Jensen-Shannon divergence (JSD) maps with a daily spatiotemporal resolution were related to sites with high catches per unit effort (CPUE) (>11 fish/103 hooks). He also concluded that a high JSD is considered to be an index of an SST front. In winter, high CPUE occurred in the vicinity of the North Subtropical Front, where SST was $15-19^{\circ} \mathrm{C}$. Monthly 
maps of joint probability density (JPD) with the optimum ranges of SST and JSD revealed that high CPUEs are located in the narrow bands with high JPD $(>50 \%)$.

\subsection{GAM:}

Venable et al (2004) provided a summary of the modelling method of Generalized Linear Models (GLMs), Generalized Additive Models (GAMs) and Generalized Linear Mixed Models (GLMMs), particularly with relation to fisheries analysis. The author additionally delineated the essential facet of model interpretation and construction thus on succeed its correct application. The analysis starts with the best models and shows the progression from GLMs to either GAMs or GLMMs. though this is often not a comprehensive review, a stress was set on topics relevant to fisheries science like transformation choices, link functions, adding model flexibility through splines, and victimization random and glued effects square measure reviewed during this study. Finally, the authors have mentioned the assorted aspects of those models and their variants and provided a read on their relative edges to fisheries analysis.

Estrada et al (2009) have analyzed the performances of computational neural networks (CNN), multiple linear regressions (MLRs) and generalized additive models (GAMs) to predict Pacific sardine landings and to analyze their relationships with environmental factors in the north area off Chile. For this purpose, several local and global environment variables and indexes (sea surface temperature, sea level, and Ekman transport index in the Chilean coast and the southern oscillation index) were considered as inputs or independent variables. Additionally, several CNN's were calibrated and validated adding the anchovy landings in the same area as model inputs. In conclusion, the results of this paper support that the quantitative prediction of sardine landings can be carried out from environmental variables, although the predictive capacity of the CNN has significantly improved if the anchovy landings are added as the input variable.

Zainuddin et al (2008) derived SST, sea surface CHL concentration (SSC), and sea surface height anomaly (SSHA) and catch data based potential habitats for albacore in the western North Pacific Ocean using the relationship between albacore fishing ground and oceanographic conditions. Empirical cumulative distribution function and high catch data analyses were used to calculate preferred ranges of the three oceanographic conditions. Results indicate that highest CPUE corresponded with areas having SST ranges $18.5^{\circ}-21.5^{\circ} \mathrm{C}$, SSC ranges $0.2-0.4 \mathrm{mg} / \mathrm{m} 3$, and SSHA ranges 5.0 to $32.2 \mathrm{~cm}$ during the winter in the period 1998- 2000. The author used these ranges to generate a simple prediction map for detecting potential fishing grounds. Statistically, to predict spatial patterns of potential albacore habitats, a combined Generalized Additive Model (GAM) Generalized Linear Model (GLM) were used. To build the model, first, a GAM was used as an exploratory tool used to identify the functional relationships between the environmental variables and CPUE. Then made parameters were derived out of these relationships using the GLM to generate a robust prediction tool. The results of this model predict the spatial pattern of the potential albacore habitat in good agreement with the simple prediction map and observation data.

Chang et al (2012) developed a habitat suitability index (HSI) \model to examine the relationships between their spatiotemporal distribution and environmental factors and to identify potential fishing grounds for the swordfish in the South Atlantic Ocean using the Taiwanese distant-water 
longline fishery data and remote-sensing oceanographic data for 1998-2007. All the environmental factors considered such as SST, mixed layer depth (MLD), sea surface height anomaly (SSHA), CHL and ocean bathymetry (BAH) - were highly significant with most of the CPUE variation explained by SST. The most optimum habitat (i.e. hotspot) was found in the areas with SST of $27^{\circ}-28^{\circ} \mathrm{C}$, SSHA of -0.05 to $0.05 \mathrm{~m}$, CHLs of $0.1-0.2 \mathrm{mg} / \mathrm{m} 3$ and BAHs of 4000 to $4500 \mathrm{~m}$. The arithmetic mean model with five environmental variables was found to be the most appropriate according to the information theory based on the evaluation of different empirical HSI models in combination with different environmental factors. The bimonthly Geographic Information System maps of the predicted HSI values were cross-validated by the observed CPUE, suggesting that the model can be used as a tool for reliable prediction of potential fishing grounds.

\subsection{DISTRIBUTION \& ECOLOGICAL PREFERENCES OF HILSA SHAD:}

Indian shad (Tenualosa ilisha) ordinarily known as Hilsa is a very prized fish in India. It is a critical transitory species in the Indo-Pacific districts. It has an extensive variety of circulation and happens in marine, estuarine and riverine conditions. The fish is found in the Persian Gulf, Red Sea, Arabian Sea, Bay of Bengal, Vietnam Sea and China Sea. The riverine living space covers the Satil Arab and the Tigris and Euphrates of Iran and Iraq, The Indus and Pakistan, the Irrawaddy of Myanmar, the streams of eastern and western India in particular the Ganga, Bhagirathi, Hooghly, Rup Narayan, Brahmaputra, Godavari, Narmada, Tapti and other beachfront streams and the Padma, Yamuna, Meghna, Karnafuly and other beachfront streams of Bangladesh. Truth be told, the Hilsa fishery in India and Bangladesh is subject to the single species viz. Tenualosa ilisha having a place with the living spaces of Indo-Gangetic and Brahmaputra stream bowls The Ganga and Brahmaputra River System is the biggest seepage where Hilsa is winning. It is broadly disseminated species under clupeid, occupying the seaside waters mostly from the Bay of Bengal and using the vast majority of the estuaries, waterways, and backwaters to breed. The present record depicts an outline of Hilsa fisheries in the Hooghly-Bhagirathi stream framework. Numerous specialists have taken a shot at Hilsa amid the most recent couple of decades, yet exhibit situation in regard of the hydrology, natural surroundings, environmental change and negative human mediations has changed to a substantial degree. Unpredictable catch of brood fish and adolescents and over-misuse have been distinguished as the most overwhelming attributes influencing the Hilsa fishery of stream framework. It is trusted that we need to attempt essential preservation measures for guaranteeing maintainable Hilsa fishery.

Hilsa sustained a lucrative fishery in the middle stretch of Ganga which declined after the commissioning of Farrakka barrage in 1972. Since 1972, the fishery has severely declined in the upstream areas and is now mainly in downstream rivers, estuaries, coastal areas and the sea. Being an anadromous species, during the southwest monsoon, Hilsa starts spawning migration to upstream and flooded regions of all rivers. Eggs are laid in freshwater and hatching takes place within $\sim 24$ hours at an average temperature of $23^{\circ} \mathrm{C}$; in about $6-10$ weeks the fry grow to about $12-20 \mathrm{~cm}$, the stage is popularly known as 'Jatka' and juveniles make their way downstream to the sea after a freshwater (Figure 05) period of 5-6 months (Mome 2007). The sea phase is about 1 year; makes Hilsa mature and undertake spawning migration towards freshwater and repeats the cycle (Haroon 1998). But some controversies are there in typical anadromous nature of Hilsa shad. 


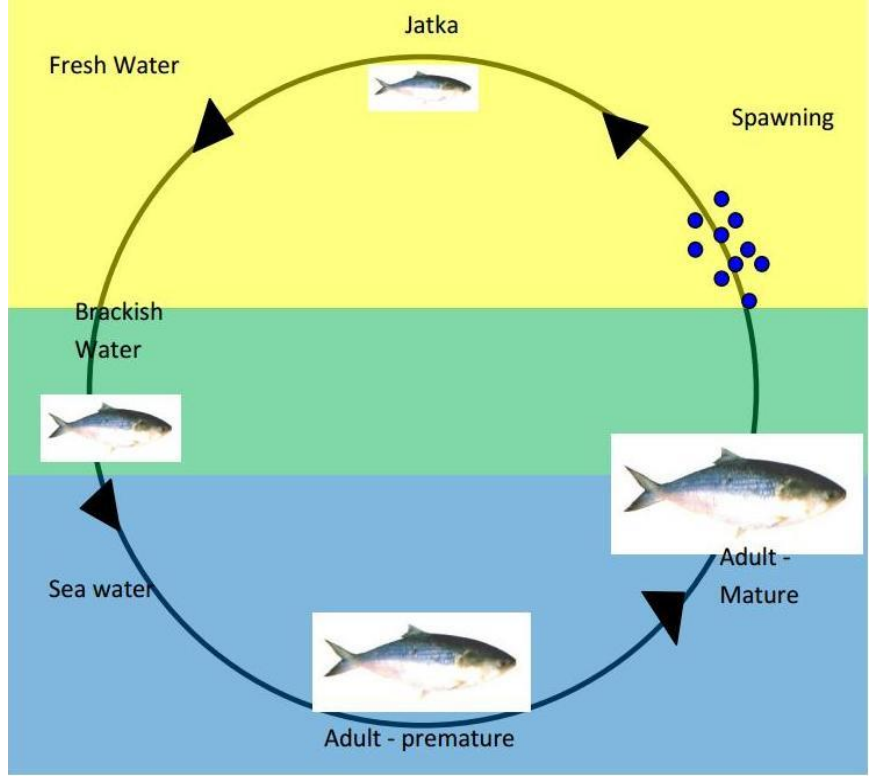

Figure Error! No text of specified style in

document.-1: Movement pattern of Tenualosa ilisha into different habitats (Mome 2007)

It is still not very clear, why Hilsa migrates towards river during breeding and which are the limiting factors influence the appearance and disappearance of Hilsa shoal along the coastal waters? Generally postbreeding in the estuary while returning to the open ocean spent fishes are open for harvest. Low water discharge from the river Ganges and associated heavy siltation, indiscriminate exploitation of juveniles, disruption of their migration routes, loss of spawning, feeding and nursery grounds and increased fishing pressure have all contributed to a decline in the catch per unit effort (CPUE) in both the marine and river Hilsa fishery.

\section{METHODS AND MATERIAL}

\subsection{FISH CATCH INFORMATION}

Fishery information utilized as a part of this investigation was gathered from fishing logbooks of different fishing boats operating in the study area. The logbook is intended to gather institutionalized data for the fish data set, the species, a number of snares and the quantity of individual fish recovered in each Fishing operation. The information from 2009-2016 are put away in a MySQL database where an inquiry is sent to pick the required datasets for examinations. Catch Per Unit Effort (CPUE) is measured dividing the total Hilsa fish catch per haul by the fishing hours of each haul.

\subsection{REGIONAL OCEAN MODELING SYSTEM (ROMS) DATA}

Major oceanic/ecological indicators, such as sea surface temperature, salinity, and chlorophyll are derived from a biophysical model configured at INCOIS using Regional Ocean Model System (ROMS). The coupled biophysical model has been validated against a wide range of observational data and has demonstrated considerable capability in reproducing marine ecosystem dynamics at synoptic and seasonal time-scales in the Indian Ocean.It is a threedimensional, free-surface, terrain-following numerical model. It simplifies equation of motion. The motivation to build a free-surface oceanic model is two-fold.

The ROMS model was set-up for the entire Indian Ocean basin from $30{ }^{\circ} \mathrm{E}$ to $120^{\circ} \mathrm{E}$ in the east-west direction and $30{ }^{\circ} \mathrm{S}$ to $30{ }^{\circ} \mathrm{N}$ in the north-south direction. The model has a horizontal resolution of $1 / 12(9.5 \mathrm{~km})$ and has 40 sigma levels in the vertical plane. The vertical profile has a resolution of $0.7 \mathrm{~m}$ near shore. Off the shelf to a bottom depth of $5000 \mathrm{~m}$, the vertical resolution ranges from $15 \mathrm{~m}$ at the surface to $500 \mathrm{~m}$ at the bottom. The bathymetry is supplied by Sindhu et al. (2007).

As the assimilation of the data available on real-time is a key component of operational ocean forecast systems, INCOIS is in the process of developing an 
ensemble Kalman Filter (EnKF) based data assimilation scheme in the ROMS setup. It is proposed to assimilate temperature and salinity profiles from the moored buoys, XBTs and the ARGO floats. By using the above ARGO floats and moored buoys, these salinity and SST data are modelled in ROMS covering most of the Indian seas and depths up to hundreds of meters.

The ROMS model was spun up for a period of 10 years from the initial state. Using Estimating the Circulation and Climate of the Ocean (ECCO) data, monthly climatological values for January are used to derive the tracer fields (temperature and salinity), Chakraborty et al. 2016

\subsection{SEA SURFACE TEMPERATURE}

This data output is in the form of NetCDF form for array analysis of locations and their corresponding SST values in various mathematical software as well as Displaying in Raster Images in GIS-based software's available.

The SST data is provided and processed in INCOIS in NetCDF format which has been dated from "01-012009 to 31-12-2015" in the region of Bay of Bengal is been collected, these figures are shown below

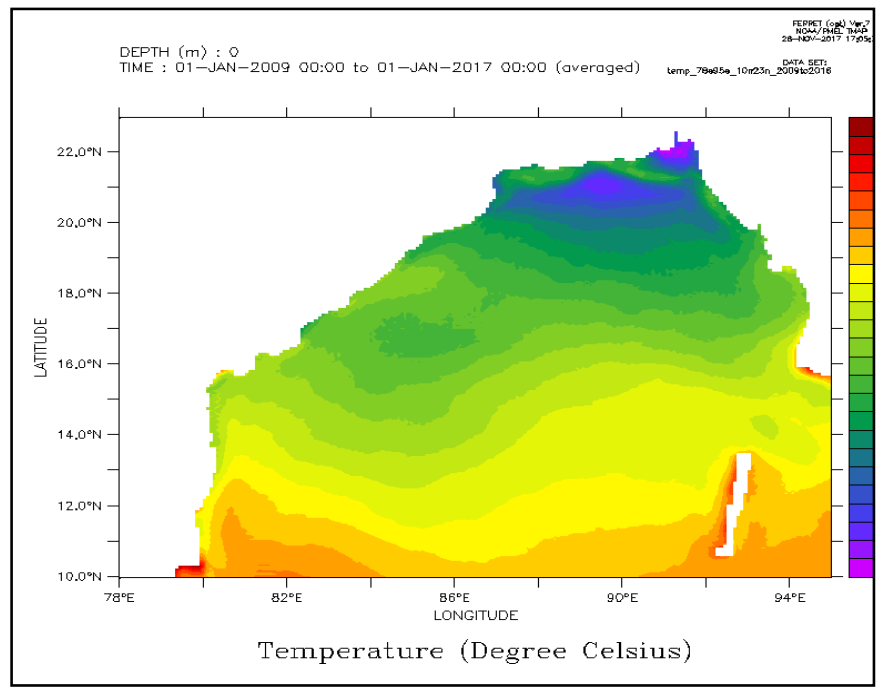

Figure 3-2: SST

\subsection{SEA SURFACE SALINITY:}

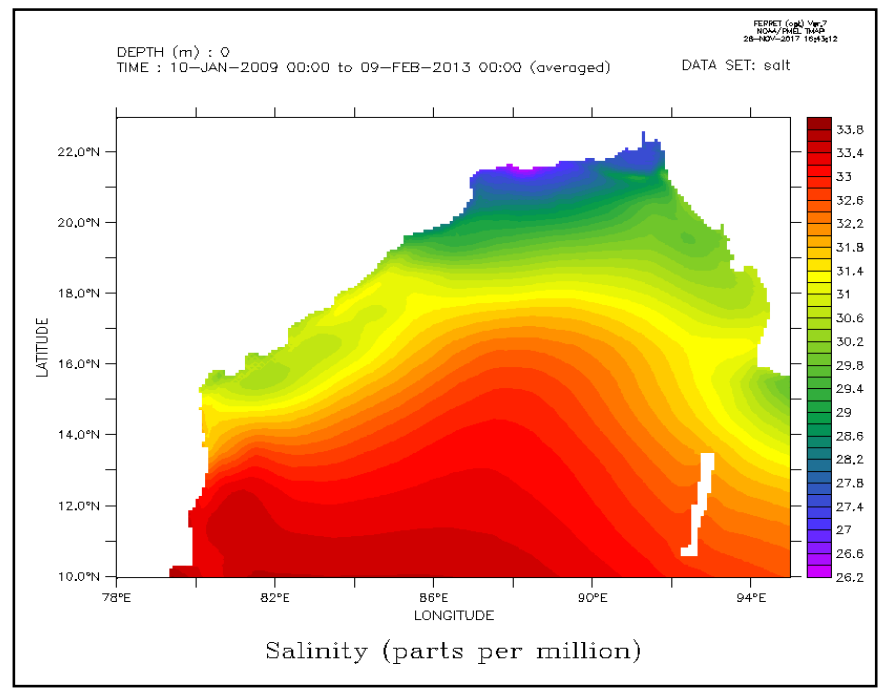

Figure 3-3: SALINITY

The ocean Surface Salinity data is provided and processed in INCOIS in NetCDF format that has been dated from "01-01-2009 to 31-12-2015" within the region of Bay of the geographical area is been collected, these figures area unit shown above.

\subsection{GENERALIZED ADDITIVE MODEL}

Using generalized additive model (GAM) catch per unit effort ( $\mathrm{kg} \mathrm{h-1)}$ is calculated as a response variable for the pre-fixed geo-locations in the study area using following equation.

$\mathrm{G}($ CPUE $)=\mathrm{a}+\mathrm{s}(\mathrm{SST})+\mathrm{s}($ Chl.$)+\mathrm{s}($ Salinity $)+\mathrm{s}($ Rainfal 1)

Where $\mathrm{g}$ is the link function, a is a constant, $\mathrm{s}($.$) is a$ spline smoothing function of the predictors

ROMS simulated sea surface temperature (SST), chlorophyll (Chl.), salinity and remotely sensed rainfall over the sea were used as predictor variables. Best-fit model was selected based on the significance of model terms, reduction in Akaike's Information Criterion and increase in cumulative deviance explained. The selected model has been used to 
predict catch per unit effort (CPUE) of Hilsa shad using predictor variables and the model was validated using a linear model. Based on geo-tagged predicted CPUE, probability maps (beta version) of suitable habitat is generated using geographic information system for further validation and fine-tuning purpose.

\section{RESULTS AND DISCUSSION}

This shows the development of monthly Hilsa shad predictive capabilities in a sustainable way. An effort has been taken to categorize essential physical, biological, and environmental signals that have a direct or indirect impact on the Hilsa shad distribution. Shad habitat has been studied from the geo-tagged fish catch data and oceanic/ecological indicators as predictor variables. For prediction, the variables are derived from a biophysical model, configured at INCOIS, using Regional Ocean Model System (ROMS) and remote sensing data. The investigation indicates the utilization of models for deciding and appreciate the spacious living space conveyance and accessibility of Hilsa in NWBOB. It is the primary Indian exertion in the Bay of Bengal wherein fishers get information is connected with modelled and satellite-inferred factors to build up an expectation framework utilizing an added substance demonstrate. Formerly, Mugo et al 2010 have attributed the habitat of skipjack tuna in the western North Pacific Sea and Rajapaksha et al. 2010 concentrated to find out the relation between oceanographic variables (from satellite) and yellowfin tuna habitat in the northeast Indian ocean using fish catch data from Sri Lankan long-line fleets using GAM.

The Fishing operations information alongside maritime covariates was isolated into a database which clarifies the maritime ecological conditions symmetric to Fishing operations in time and space. Information from this Master Sheet was utilized to create GAM models that give more data on the natural surroundings reasonableness of the Hilsa shad species.

\subsection{GAM PLOTS:}

Below figures are generated using GAM and the plots indicate distribution range with 95\% confidence level. The narrow confidence level indicates high relevance among each other and wider confidence level indicates less relevance.

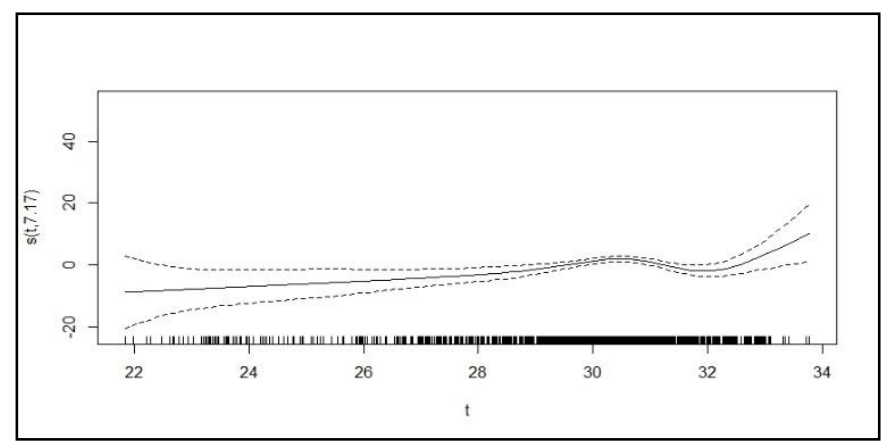

Figure 4-4 : Plot showing CPUE variation with Temperature in "best-fit model"

Based on the GAM plots \& AIC values, the favorable oceanographic parameters for Hilsa shad aggregation off North West Bay of Bengal were characterized by; SST of $29-32{ }^{\circ} \mathrm{C}$ in the above Plot Solid dashed line in with lines converging gives 'best fit' region plots indicates the $95 \%$ confidence intervals and most influential region on Response Predicted CPUE.

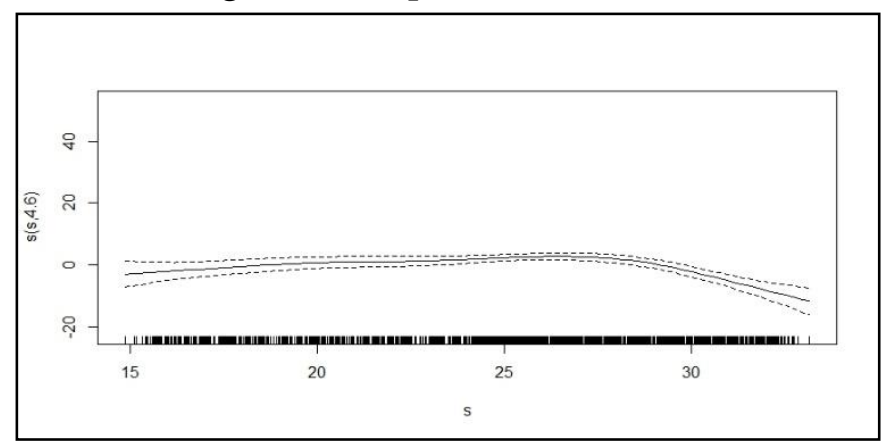

Figure 4-5: Plot Showing CPUE variation with Sea Surface Salinity in Best-Fit Model

Based on the GAM plots \& AIC values, the favorable oceanographic parameters for Hilsa shad aggregation off North West Bay of Bengal were characterized by; 
Sea Surface Salinity is $25-30$ in the above Plot Solid dashed line in with lines converging gives 'best fit' region plots indicates the $95 \%$ confidence intervals and most influential region on Response Predicted CPUE along with other three Predictor terms salinity has third best influence on Response CPUE.

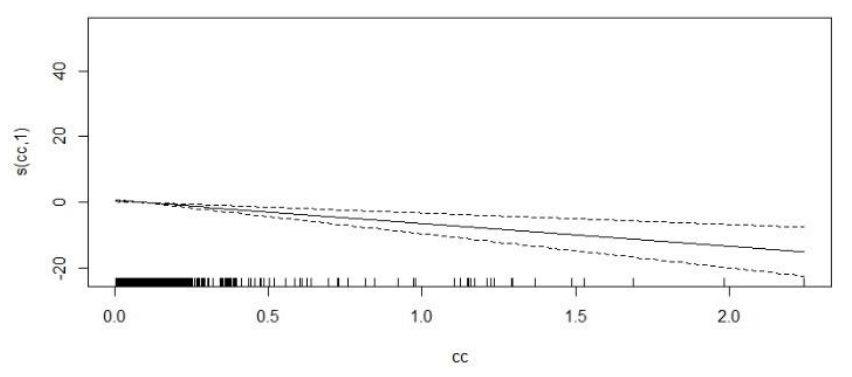

Figure 4-6 : Plot showing CPUE variation with chlorophyll in "best-fit model"

The favorable oceanographic parameters for Hilsa shad aggregation off North West Bay of Bengal were characterized by; Chl-a of range $0.05-0.5 \mathrm{mg} / \mathrm{m}^{3}$ in the above Plot is greatly affecting the GAM-derived Response of Predicted Solid dashed line in with lines converging gives 'best fit' region plots indicates the 95\% confidence intervals and most influential region on Response Predicted CPUE

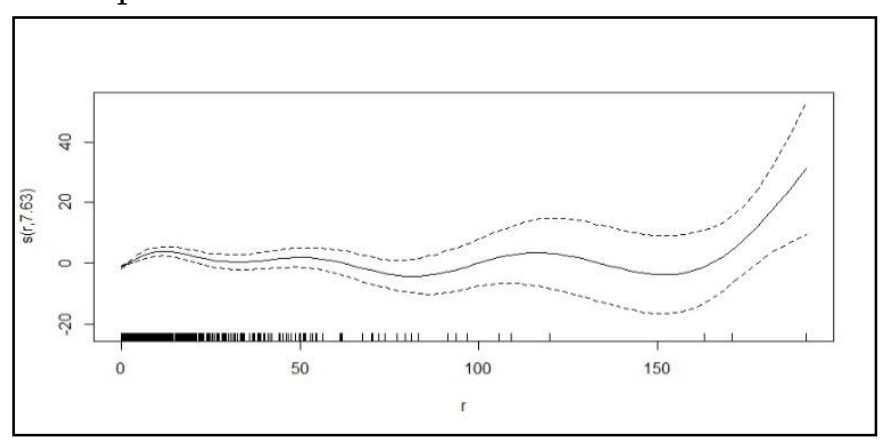

Figure 4-7 : Plot showing cpue variation with TRMM Rainfall in "best-fit model"

From Table 2 as mentioned below containing GAMDerived AIC values \& \% Deviance Explained the parameter which is Satellite-derived TRMMRainfall $(\mathrm{mm} / \mathrm{hr})$ is having highest weightage and great infulence on Hilsa Shad migration and availabilty in Monsoon Season and time period having good rainfall, from above Plot we can clearly see the influence of TRMM on Response Predicted CPUE as in the range of $0-50 \mathrm{~mm} / \mathrm{hr}$ rainfall has best influence of $95 \%$ confidence intervals as lines are converging and 50-100 mm/hr has medium and above $100 \mathrm{~mm} / \mathrm{hr}$ has minimal influence, hence the most important predictor in this modelling is TRMMRainfall

Table 4-1 : GAM-derived deviances and AIC values

\begin{tabular}{|c|c|c|}
\hline Hilsa & $\begin{array}{l}\% \\
\text { deviance_explained }\end{array}$ & AIC \\
\hline \multicolumn{3}{|l|}{ ONE VARIABLE } \\
\hline Rainfall & 4.85 & $\begin{array}{l}14729.6 \\
0\end{array}$ \\
\hline SST & 4.86 & $\begin{array}{l}15550.0 \\
0\end{array}$ \\
\hline Salinity & 1.69 & $\begin{array}{l}15610.2 \\
0\end{array}$ \\
\hline Chlorophyll & 1.69 & $\begin{array}{l}15617.8 \\
8\end{array}$ \\
\hline \multicolumn{3}{|l|}{ TWO VARIABLES } \\
\hline SST+Rainfall & 7.86 & $\begin{array}{l}14527.4 \\
0\end{array}$ \\
\hline Salinity+Rainfall & 6.00 & $\begin{array}{l}14556.8 \\
6 \\
\end{array}$ \\
\hline SST+Salinity & 6.37 & $\begin{array}{l}15530.0 \\
0\end{array}$ \\
\hline SST+Chlorophyll & 6.17 & $\begin{array}{l}15536.1 \\
7\end{array}$ \\
\hline Salinity+Chlorophyll & 3.18 & $\begin{array}{l}15593.1 \\
0\end{array}$ \\
\hline \multicolumn{3}{|l|}{ THREE VARIABLES } \\
\hline SST+Salinity+Rainfall & 9.37 & $\begin{array}{l}14505.0 \\
0\end{array}$ \\
\hline Rainfall+Chlorophyll+SST & 8.95 & $\begin{array}{l}14510.0 \\
0\end{array}$ \\
\hline SST+Salinity+Chlorophyll & 7.63 & $\begin{array}{l}15512.0 \\
0 \\
\end{array}$ \\
\hline ALL VARIABLES & & \\
\hline $\begin{array}{l}\text { SST+Salinity+Chlorophyll+ } \\
\text { Rainfall }\end{array}$ & 10.4 & $\begin{array}{l}14486.4 \\
4 \\
\end{array}$ \\
\hline
\end{tabular}

Above GAM derived variables along with those AIC values of all possible combinations in increasing order of parameters with Best Fitted models have been generated on the yearly scale and placed in the 
above Table. The model explained the influence of each parameter on CPUE through the AIC values. The individual parameter with least AIC value will be in the top priority which has a huge impact on the CPUE. In case of this study, rainfall is on the top with AIC value of 14729.60 . The second one is SST that had AIC value of 15550 . The third one will be salinity with AIC value of 15610.20 . And, the least prior parameter is chlorophyll with AIC value of 15617.88. GAM model result for the Hilsa species is depicted in the last part of the table considering all parameters, SST, salinity, chlorophyll, and rainfall.

The model explained $10.4 \%$ deviance considering all variables. The selected model had the lowest AIC value of 14486.44. Using the same model monthly CPUE for September to December 2016 has been calculated in different predetermined points falling in the study area. The excel data, containing CPUE of predetermined points are incorporated in the GIS platform using ArcGIS software and exported into point layer form. Using point to raster conversion tool the point data converted into a raster layer. The catch information brings to a range of 0 to 1 by taking $\log _{10}$ (CPUE) and stored as an additional column in the attribute table. Raster layer is generated on the basis of logarithmic information of catch data. The best way to represent data with High, Medium and Low catch data information using GIS Software is thematic map representation. The value between 1 to 0.6 is considered as High Catch and the value between 0.6 to 0.4 is considered as medium catch and the value below 0.4 is considered as a low catch.

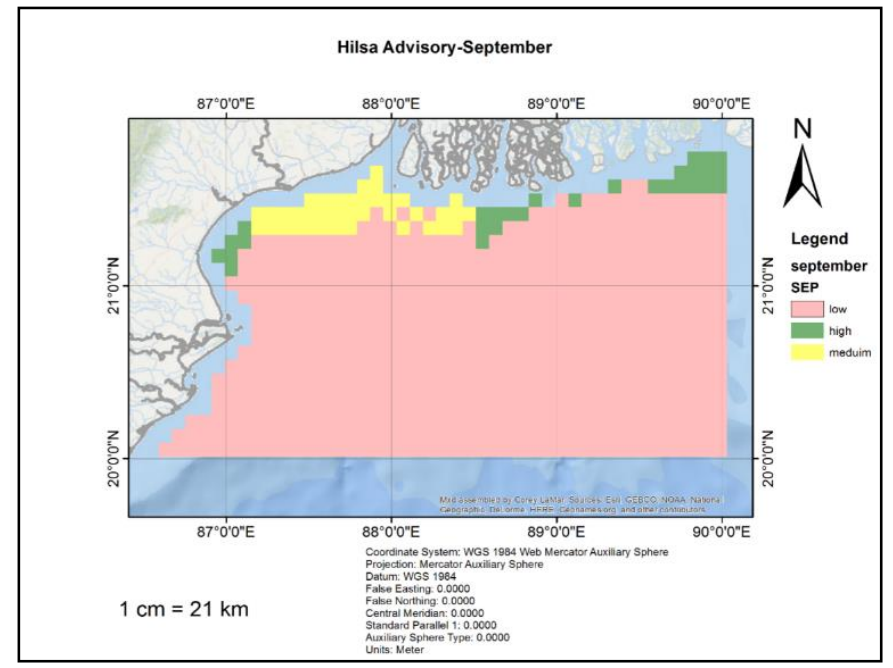

Figure 4-8: Habitat Suitability map (September)

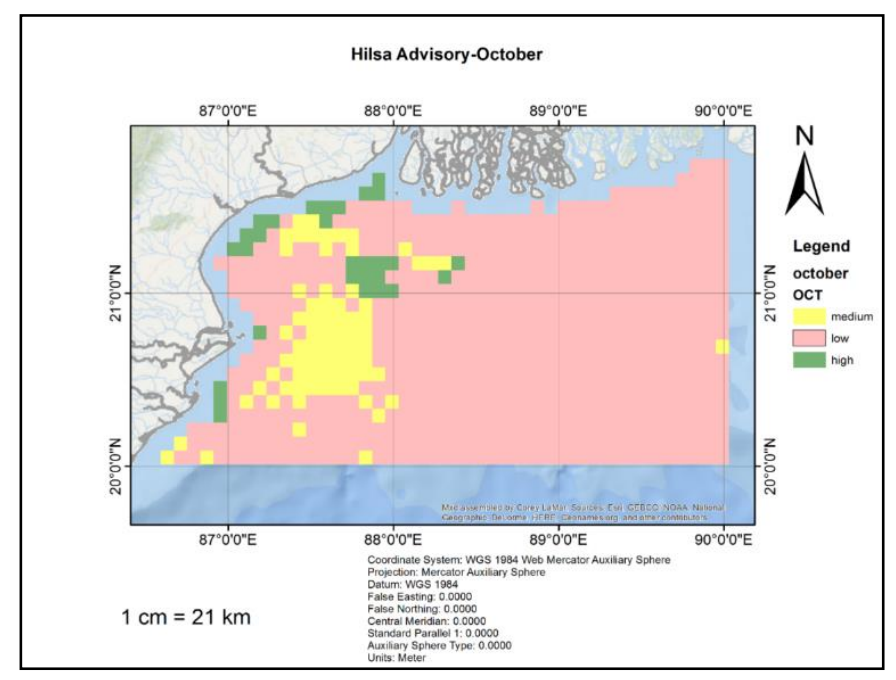

Figure 4-9 : Habitat Suitability map (October)

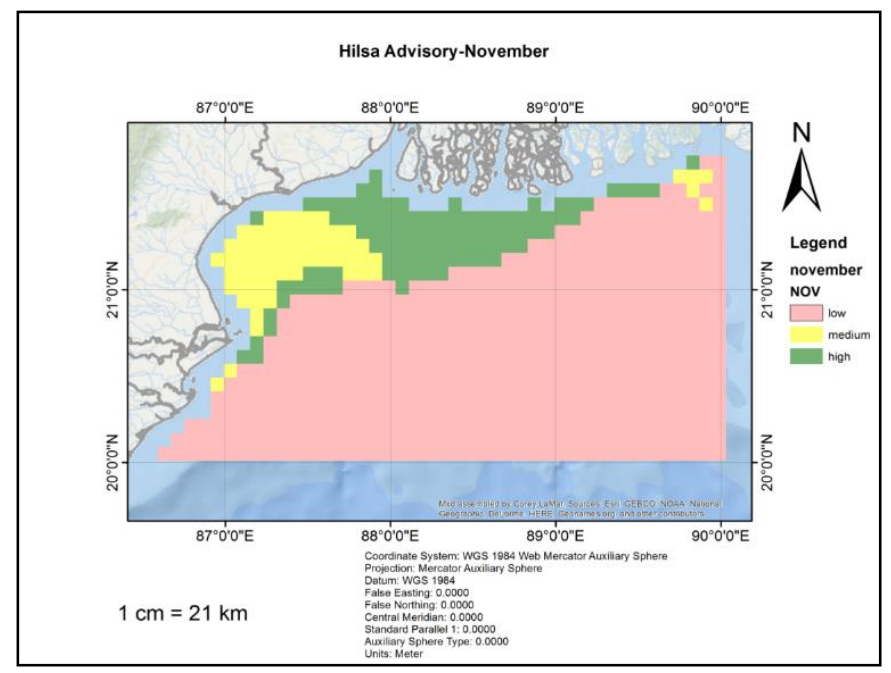

Figure 4-10 : Habitat Suitability map (November) 


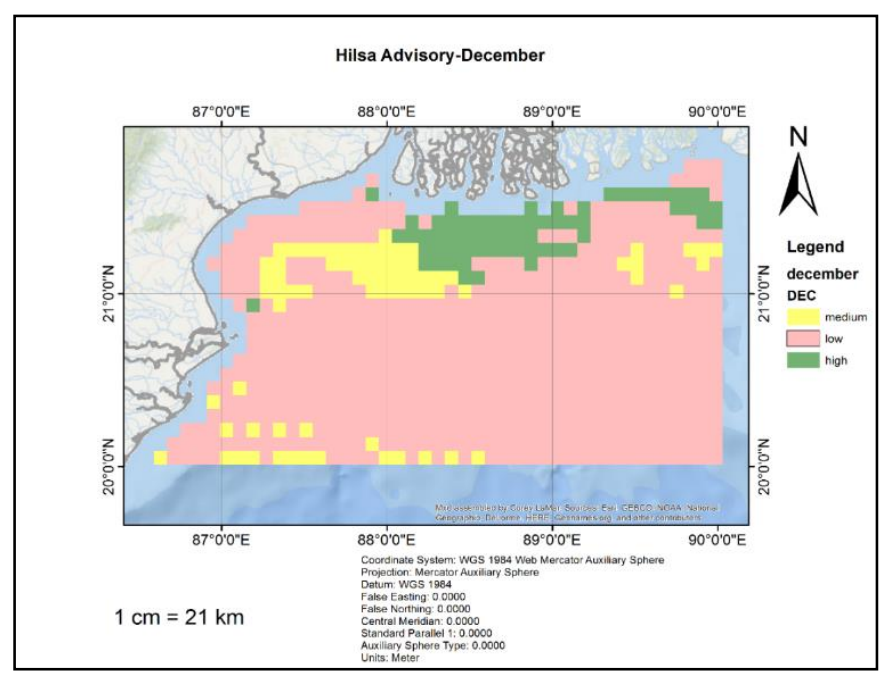

Figure 4-11 : Habitat Suitability map (December)

\section{v. CONCLUSION}

This study characterized the suitable forecast regions for HILSA distribution in along the Coast of NORTHWEST BAY OF BENGAL using fishing operations data and satellite-derived biophysical variables. Habitat and food indicators were linked with satellite-derived biochemical and physical parameters. GAM model was applied to predict HILSA distribution in the North West Bay Of Bengal. Model outputs are in good coherence with the species response to the environmental condition. A Good Percentage deviance was explained by the BEST FIT model. Based on AIC and deviance metrics GAM was trained on several combinations of input variables to generate the best fit model. But it needs a minimum number of adequate points to validate the model and to forecast HILSA CPUE. Classification results from the GAM model used to forecast the levels of CPUE as High, Medium \& Low. Model results were validated through in-situ data.

The model generated from GAM analysis indicated a good match with higher CPUE points in the potential predicted areas in the validation exercise. Factors affecting marine fishery species can be multiple and hence, take into account as many as possible can provide a better understanding of their interactions with the environment. Although the present study provides some good preliminary results, implementation of a near real-time dynamic model with the input of available oceanographic variables may further improve the accuracy of predicting fish abundance areas.

\section{REFERENCES}

[1]. Anon, 2012, Report of Department of Fisheries, Government of West Bengal.

[2]. R.A. Arnone, Satellite-derived colourtemperature relationship within the Alboran ocean, Remote Sensing of setting twenty-three (1987), pp. 417-437

[3]. Chang Y, Sun C, Chen Y, Yeh S, Dinardo G. 2012. Habitat suitability analysis and identification of potential fishing grounds for swordfish, Xiphias gladius, in the South Atlantic Ocean. Int J Remote Sens. 33:75237541.

[4]. Yen K, Lu H, Chang Y, Lee M. 2012. Using remote-sensing data to detect habitat suitability for yellowfin tuna in the western and Central Pacific Ocean.Int J Remote Sens. 33:7507-7522.

[5]. S. B. Choudhury, B. Jena, M. V. Rao, K. H. Rao, V. S. Somvanshi, D. K. Gulati \& S. K. Sahu International Journal of Remote Sensing Vol. 28, Iss. 12,2007 Validation of integrated Potential fishing Zone (IPFZ) forecast using satellite-based chlorophyll and sea surface temperature along the east coast of India.

[6]. Pacific sardine (Sardinops sagax, Jenyns 1842) landings prediction. A neural network ecosystemic approach Juan Carlos GutiérrezEstradaa EleuterioYáñezbInmaculadaPulidoCalvoaClaudioSilvabFranciscoPlazabCinthyaBó rquezb 2009

[7]. Hosoda, Kohtaro \& Kawamura, Hiroshi \& Lan, Kuo-Wei \& Shimada, Teruhisa \& Sakaida, 
Futoki. (2012). Temporal Scale of Sea Surface Temperature Fronts Revealed by Microwave Observations. IEEE Geosci. Remote Sensing Lett.. 9. 3-7. 10.1109/LGRS.2011.2158512.

[8]. Hastie T, Tibshirani R. 1986. Generalized additive models. Stat Sci. 1:297-310

[9]. Haroon, Y, 1998. Hilsa shad: Fish for the teeming millions, new management alternatives needed for the hilsa young. Shad Journal, 3:7

[10]. Laurs R.M., Fielder, P. C. and Montgomery, D.R. (1984). Albacore tuna catch distribution relative to environmental features observed from satellite. Deep Sea Research, 31:1085-1099

[11]. Mugo, R., S. Saitoh, A. Nihira, and T. Kuroyama. 2010. "Habitat Characteristics of Skipjack Tuna (Katsuwonus Pelamis) in the Western North Pacific: A Remote Sensing Perspective." Fisheries Oceanography 19 (5): 382-396. doi:10.1111/j.13652419.2010.00552.x.[Crossref], [Web of Science ®], [Google Scholar]

[12]. Mome M.A., 2007. The potential of the artisanal hilsa fishery in Bangladesh: an economically efficient fisheries Policy. The Fisheries Training Programme, The United Nations University, Iceland.

[13]. Rajapaksha JK, Nishida T, Samarakoon L. 2010. Environmental preferences of yellowfin tuna (Thunnus albacores) in the Northeast Indian Ocean: an application of remote sensing data to longline catches. Report of the Sixth Session of the IOTC. Victoria: Working Party on Ecosystems and bycatch. (Working Party on Ecosystems and Bycatch; IOTC-2010-WPTT43, 27-30 October 2010).

[14]. R Core Team. 2014. R: A language and environment for statistical computing. $R$ foundation for statistical computing, Vienna,
Austria; [2014 Dec 11]. Available from: http://www.R-project.org/

[15]. Solanki HU, Mankodi PC, Nayak SR, Somvanshi VS. 2005. Evaluation of remotesensing-based potential fishing zones (PFZs) forecast methodology. Cont Shelf Res. 25:21632173

[16]. Sanchez P, Demestre M, Recasens L, Maynou F, Martin P. 2008. Combining GIS and GAMs to identify potential habitats of squid Loligo vulgaris in the Northwestern Mediterranean.Hydrobiologia. 612:91-98.

[17]. Applications of a generalized additive model (GAM) to satellite-derived variables and fishery data for prediction of fishery resources distributions in the Arabian Sea. H. U. Solanki, DhyeyBhatpuria\&PrakashChauhanGeocarto International Published Online: 13 Jan 2016

[18]. Predicting potential fishing zones of Japanese common squid (Todarodespacificus) using remotely sensed images in coastal waters of south-western Hokkaido, Japan Xun Zhang, Sei-Ichi Saitoh\& Toru Hirawake International Journal of Remote SensingXun Zhang, Sei-Ichi Saitoh\& Toru Hirawake Pages 6129-6146 | Published online: 11 Dec 2016

[19]. Venables, W.N., Dichmont, C.M., 2004. A generalized linear model for catch allocation: an example of Australia's Northern Prawn Fishery. Fish. Res. 70, 405-422.

[20]. Wood S. 2006. Generalized additive models: an introduction to R. Chapman and Hall/CRC Boca Raton (FL): CRC Press. ISBN: 978-158488-474-3.

[21]. Yamanaka, I., Ito, S., Niwa, K., Tanabe, R., Yabuta, Y., \&Chikuni, S. (1988).The fisheries forecasting system in Japan for coastal pelagic fish. FAO Fisheries Technical Paper, 301 (72 pp.) stem in Japan for coastal pelagic fish. FAO Fisheries Technical Paper, 301 (72 pp.) 
[22]. Zainuddin M, Saitoh K, Saitoh S. 2008. Albacore (Thunnusalalunga) fishing ground in relation to oceanographic conditions in the western North Pacific Ocean using remotely sensed satellite data. Fish Oceanography. 17:61-73.

[23]. Zainuddin, Mukti. (2011). SKIPJACK TUNA IN RELATION TO SEA SURFACE TEMPERATURE AND CHLOROPHYLL-A CONCENTRATION OF BONE BAY USING REMOTELY SENSED SATELLITE DATA. Jurnal Ilmu dan Teknologi Kelautan Tropics. 3.. 10.28930/jitkt.v3i1.7837.

\section{Cite this article as :}

Bandanadam Swathi, Swarnalatha. V, Venkatesh Jogu, "The Use of Generalized Additive Model (GAM) To Assess Fish Abundance and Spatial Occupancy in North-West Bay of Bengal", International Journal of Scientific Research in Science and Technology (IJSRST), Online ISSN : 2395-602X, Print ISSN : 2395-6011, Volume 6 Issue 3, pp. 17-28, May-June 2019. Available at doi : https://doi.org/10.32628/IJSRST19632 Journal URL : http://ijsrst.com/IJSRST19632 